\title{
Construction of Laboratory Platform based on Cloud Computing
}

\author{
Yueping $\mathrm{Wu}^{1}$, Yinchun Yang ${ }^{2}$ \\ ${ }^{12}$ Higher Vocational (International) College, Shanghai Second Polytechnic University, Shanghai \\ 201209, China
}

Keywords: Cloud computing, virtualization, Remote Desktop Protocol, server, service.

\begin{abstract}
In view of the problem which the present laboratory management cost is too high, resource waste severely, data is not unified, it was proposed that construction of laboratory platform based on cloud computing, the plan aims to construct the virtual pool of resources, and ultimately achieve a unified cloud platform, which build a sharing, dynamic, autonomous service, data center extended with the need, to provide a new IT resource supply mode for the teaching and scientific research. In this mode, teachers and students can get their desktop computing resources in any place at any time. Another important application about cloud computing in the laboratory is the construction of the virtual service, can deploy a server for storage, examination and race in the room in a few minutes by VSphere. Specific benefits are shown in table after the implementation of server virtualization.
\end{abstract}

\section{The Concept of Cloud Computing}

The concept of Cloud computing [1,2] is concerned, since it was proposed, and then the vigorous development. In 2008 April, Google released the application service engine (Google App Engine), developers regardless of project size can create applications from the GAE platform. While Microsoft Company introduced the first "cloud computing" in the product after the Live Mesh platform, and in 2008October launched to "cloud computing" as the core of Windows Azure services, Can help developers across the cloud and the next generation of professional data center application, In the $\mathrm{PC}$, Web, mobile phones and other terminal to create the perfect user experience .Thus, "cloud computing" is becoming the development direction of next generation Internet technology.

Cloud computing is based on the Internet related services increasing, using and delivery mode, usually via the Internet provides dynamically scalable and often virtualized resources.

Figure 1 is a typical cloud computing architecture schematic diagram, the user terminal equipment through the network access to the cloud, can carry on the corresponding data computation and storage operation. It can be seen from Figure 1, user management is the main entrance to the user terminal access to the cloud, the cloud computing service providers go to control management with user which access to the cloud through this layer. Service layer will integrate various resources of clouds, according to the needs of users, provide the corresponding application services (such as infrastructure as a service, platform as a service, software as a service etc. [3]).Virtual resource layer is to integrate various resources through virtualization management, for the service layer allocation. The bottom of the cloud computing architecture is physical resource layer, which is the foundation of cloud computing platforms, deploying a variety of real physical devices. By introducing the virtualization technology, cloud computing users are free from the trivial, complex computer resource management, but also is to ensure the confidentiality and integrity of user data, and greatly improve the utilization rate of all kinds of resources.

Cloud Computing Service Type. Cloud computing services based on its hierarchical structure can be divided into three categories. The bottom is the infrastructure as a Service, Mainly to provide users with basic computer equipment and facilities in the form of services, including server cluster, computer hardware, network equipment, storage equipment and other resources. The second layer is platform as a Service, mainly to provide users with server platform, middleware platform and hardware platform, the users can develop the applications or content on the platform, and to provide services with other users, a typical such as Google App Engine, Windows Azure cloud platform. 
The top is a software as a service, cloud computing service and content providers will centrally deploy demanded application software in the server group, through the Internet to provide users with services, at this point, the end user does not need to download or maintain any application software, only according to their own needs to apply for cloudy computing center and register, after the successful application, you can pay the corresponding fees for cloudy computing center, to use the relevant application software, and then customize and develop personalized application on this basis, a typical such as Gmail, Live Service, etc.

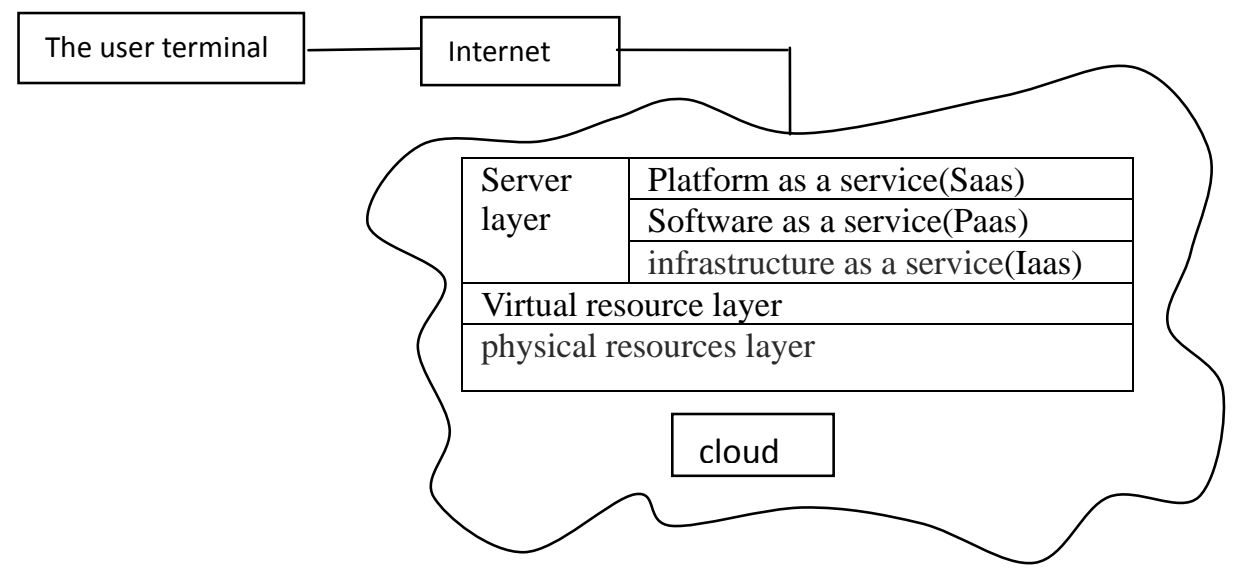

Figure 1 Cloud computing architecture schematic diagram

The Characteristics of Cloud Computing. Compared with the traditional application model, cloud computing platform has the following features:

a.The Virtualization Technology

Cloud computing platform through virtualization technology provides the various hardware resources, calculate capacity, network resources and information resources, regardless of the users in where, and how the local environment, need only through a web browser can use these resources, realize the application and demand.

b. Service Flexibility in Customizing

According to the user needs cloud computing platform deploy the resources and services, users only need to focus on demand, does not need to care about where is resource, how to obtain, cloud computing platform, cloud computing platform will be returned the results as a service to the customer, if the user needs to change or add demand, only need to modify or add customized service.

c.High Cost Performance

The user always pursuit of low prices, excellent service, and the cloud computing happened to meet this requirement, users only need to pay service fees, do not need to spend a lot of cost to purchase software and hardware equipment.

d.Safe and Reliable

Data security is very concerned about the user, and the cloud data is stored on the server, manage professionally, through strict rights management strategy can help users at the same time of data sharing not to worry about data corruption, viruses etc.

\section{Cloud Computing in the Laboratory}

The Use of Cloud Computing in the Laboratory. In 2007 October, Google and IBM jointly announced the promotion of "cloud computing", including Carnegie Mellon University, Stanford University, Massachusetts Institute of Technology, California University at Berkeley, all participated in the project, jointly study the development of cloud computing and technology, and provides the hardware and software equipment and computing techniques for laboratory support. Laboratory using cloud computing support, to further improve the resource construction and service ability is representing the general trend. 


\section{The Advantages of Cloud Computing in the University Laboratory.}

a.More Reasonable Sharing of Resources

Cloud computing technology in the lab combined to form a whole, sharing vast infrastructure and resources, without frequent replacement of hardware equipment, thereby reduce the hardware cost of the university laboratory, and the effect of resource sharing [4].

b. More Efficient Maintenance Operation of Software and Hardware

After university Laboratory accessing cloud computing platform, both the server hardware maintenance and the software update, maintenance and other content, will have a cloud computing service provider to offer professional service, users need only a browser running could enjoy the convenience features of cloud computing.

c.More Safe and Reliable Data Storage

The university laboratory has a number of important resources and data storage, processing and use, and, the data storage, which are caused the serious threat by the rampant computer virus, network attack. At the same time laboratories of colleges and universities need to purchase the database server to meetthe storage requirements of growing laboratory resources data, and after cloud computing services, all of these can provide services by the cloud computing service providers.

d. More Efficient Service and Resource Utilization

Through optimization application of cloud computing technology, a wide range of resources and services in the laboratory can be more convenient for the users whenever and wherever, learning activities to master more by learning themselves rather than university greatly optimized the learning environment, help students' self-study.

\section{Construction of Laboratory Information Platform based on Cloud Computing}

In order to improve the future application and the overall performance, reliability and flexibility of the teaching, scientific research platform, the plan aims to construct the virtual pool of resources, and ultimately achieves a unified cloud platform, which build a sharing, dynamic, autonomous service, extended data center with the need, to provide a new IT resource supply mode for the teaching and scientific research. In this mode, teachers and students can get their desktop computing resources in any place, centralized data center can also manage and dynamically allocate, deploy, configure, recover resources, can also support automatic installation of software and application in the future, so as to realize the rapid, efficient, dynamic optimizational scientific research, teaching application, development platform and computing resource allocation.

Construction Project and Architecture Plan. The information construction project is divided into three stages: The first stage that achieve part of desktop virtualization as a pilot, can provide more than 200 Windows desktops (50 concurrent users), 50 virtualization environment of Linux desktop, while providing a small cloud platform for future server consolidation. The second stage that can expand the scale of desktop virtualization on this basis, meet 2000 teachers and students, concurrent 400-500 Windows Desktop needs, at the same time, the Linux Desktops reach 100, build the teaching cloud platform. Another teacher's teaching system can be also included in the desktop cloud, teachers can land own desktop to get the required information on any occasion. The third stage that perfect desktop cloud system, complete to migrate the existing business to virtualization platform, at the same time, build high-performance computing cloud platform to integrate the scientific calculation of the college. The three stages are continuous, and each stage ensures an ascension to complete school information, and lays a foundation for the next phase of the project.

According to the actual application situation, it is recommended that the scheme deployment take different architecture scheme for different business:

For core or not virtualization systems still use the physical machine which run directly the original operating system and database, in order to guarantee the reliability of the server and application, it's suggest that the business is still able to provide in the case of unplanned and planned downtime situation in the independent dual cluster way. 
For many application servers, using VMware server virtualization technology, apply some of services integrated into 2 sets of 4 road 6 or 8 nuclear physical machine, realizes the virtualization of physical server. Overall integration refer to the host operation ability at about 8:1.

In aspect of data storage, in addition to considering as the traditional way to put the database data on the SAN storage display, at the same time the package file virtual architecture suite produced are stored in the SAN storage array. Through the SAN storage structure of sharing, can maximize the advantages of virtual architecture, migrate online to the running virtual machine (VMware VMotion), dynamic resources management (VMware DRS) and integrated backup based on snapshot technology Lan Free of the virtual machine (VMware VCB)etc, and to provide scalability for future disaster recovery and lay the foundation.

Project Operation Mode. RDP (Remote Desktop Protocol) is a terminal services network communication protocol building on the Windows operating system. It uses a typical $\mathrm{C} / \mathrm{S}$ architecture, and consists of two parts: the client running on a remote device and the terminal server [5].

It is the purpose that the Windows screen display data information on the terminal server is passed smoothly to the client through RDP protocol [6]. The RDP protocol allows users to use safely all applications, files and network resources on the remote computer, as I am in front of the remote computer. Not only that, any applications users running locally still can run normally by using a remote desktop.

Based on the application of cloud computing service mode is the concrete application of cloud computing. The basic design idea is: to provide users with remote desktop service in the cloud, the service provider in the data center server runs on the operating system and application software according to user's requirements, and then the operating desktop view in the form of image will be transmitted to the terminal equipment by the remote desktop protocol (R D P). At the same time, the server collects and processes all kinds of input terminal information, and updates the content of the desktop view at any time according to the specific situation of dealing.

Since all computations are performed on the server, so the terminal equipment requirements are relatively low, only the client access the cloud in the network, can carry out the corresponding operation and enjoy the corresponding service; Use this application service providing mode, through some equipments access the personal desktop system on the network and operate accordingly in any places and at any times. For example, teachers in the classroom teach computer professional courses, in different classroom or different semester, do not need install specially corresponding teaching software, and only need to access the own remote desktop, equivalent to carrying a mobile computer with the necessary software.

Another important application about cloud computing in the laboratory is the construction of the virtual service [7]. each laboratory will need to spend a certain amount of manpower, financial resources maintaining the running of servers in each semester, now the server data migrates to the cloud, to build a unified template according to the need, each room does not need to set up a server, but only need to apply, can deploy a server for storage, examination and race in the room in a few minutes by VSphere.

Benefit Comparison before and after Implementation. After the implementation of server virtualization, the direct effect is that the existing more than 10 physical servers integrate for 2 sets, the number of servers, network equipment, racks, air conditioning power in the computer room, energy consumption other peripherals and IT equipment supporting will be substantially reduced, the environment of computer room becomes more manageable. After the original business migrate to the virtual data center, the business will have high availability virtualization technology bringing, to avoid the single point of failure. Specific benefits are shown in table 1.

Table 1. Comparison before and after implementation

\begin{tabular}{|l|l|l|}
\hline Item & $\begin{array}{l}\text { The traditional physical machine } \\
\text { architecture }\end{array}$ & VMmare virtual architecture \\
\hline The number of server & $10+$ & 2 \\
\hline
\end{tabular}




\begin{tabular}{|l|l|l|}
\hline The utilization of CPU resources & $15 \%$ & $60 \%$ \\
\hline The utilization of memory & $30 \%$ & $75 \%$ \\
\hline The utilization of network bandwidth & $30 \%$ & $70 \%$ \\
\hline Outage update time & One hour & No downtime \\
\hline Unexpected downtime & Several hours (uncontrolled) & No downtime \\
\hline Storage migration time & Two days & Four hours \\
\hline System restore time & Three hours & Three minutes \\
\hline A single point of failure & have & no \\
\hline HA high availability cluster function & no & Built-in \\
\hline Fault tolerant server function & no & support \\
\hline Dynamic resource optimization & no & support \\
\hline Operating system deployment time & Five hours & Half hour \\
\hline Upgrade the hardware way & Outage update & Business automatic migration \\
\hline Early application compatibility & no & Built-in \\
\hline Patch management & The third party & Built-in \\
\hline Management interface & Multiple scattered & A centralized \\
\hline
\end{tabular}

\section{Conclusion}

Application services based on cloud computing model make calculation, storage and other application service requests to users put forward to be processed in the cloud, make full use of the resource of the server, reduce the terminal performance requirements. no matter when and where, User as long as uses the terminal device accessing network, can easily enjoy the corresponding real-time application service .It's believe that the continuous development and mature of cloud computing technology will better promote the process of educational information. At present, the application of cloud computing in university is still at the initial stage, and believe that the cloud computing is becoming more and more attention, the information development of our university laboratory will be more on a stage.

\section{References}

[1] Buyya, R., Yeo, C., Venugopal, S., Broberga, J., Brandic, I.: Cloud computing and emerging IT platforms: vision, hype, and reality for delivering computing as the 5th utility. Future Generation Computer Systems 25, 599-616 (2009)

[2] Armbrust, M., Fox, A., Griffith, R., Joseph, A., Katz, R., Konwinski, A., Lee, G., Patterson, D., Rabkin, A., Stoica, I., Zaharia, M.: A view of cloud Computing. Communications of the ACM 53, 50-58 (2010)

[3] ZhangJianxun, GuZhimin, Zheng Chao, Survey of research progress on cloud computing [ J ],Application Research of Computers. 2010.27(2):429-433

[4] Gao Wushi, Research on virtualization technology and cloud computing application (J), Applied Mechanics and Materials,(VMEIT 2014)2014. 543-547: 3598-3601

[5] Wang Shuai, Application Service Provider Model Based on Cloud Computing(J), Journal of Zhongyuan University of Technology, 2013.24(4):54-58

[6] Liu Wei, An Application Service Pushing Model based on RDP(D),Guangzhou: Zhong Shan University,2010.

[7] Wu Yueping, Zhengjian, Duyi, Building of Virtual Server in Computer Laboratory,Lecture Notes in Electrical Engineering Journal(ICISME 2012)2013.219:373-379. 